\title{
Modelagem Matemática e Algoritmo de Programação Associados à Simulação Matemática do Volume de um Tanque
}

\author{
João Socorro Pinheiro Ferreira1 1 ic \\ Universidade Federal do Amapá (UNIFAP), Departamento de Ciências Exatas e Tecnológicas, \\ Coordenação de Licenciatura em Matemática, Macapá, Amapá, Brasil.
}

\begin{abstract}
Resumo: Este artigo científico tem como objetivo apresentar os resultados de um relato de experiência de ações pedagógicas para a resolução de uma situação-problema sobre o volume de um líquido variando no interior de um tanque cilíndrico de base cônica, que engloba modelagem matemática e linguagem de programação, especificamente no que diz respeito à possibilidade de se inter-relacionar duas técnicas de ensino com uma finalidade comum: modelagem matemática e algoritmo de programação associadas à simulação matemática. Para atingir os objetivos, primeiramente modelamos matematicamente a situação-problema proposta com funções de duas variáveis reais positivas e três sentenças, mediante procedimentos matemáticos e posteriormente simulamos matematicamente os valores do raio $\mathrm{R}$ e da profundidade " $\mathrm{d}$ " do líquido no interior e "exterior" do tanque - diretamente no programa computacional desenvolvido. Os resultados dos estudos matemáticos e computacionais mostraram-se satisfatórios aos objetivos propostos aos estudos, isto porque, os valores obtidos por métodos distintos apresentaram os mesmos números reais.
\end{abstract}

Palavras-Chave: BNCC. MATLAB. VISUALG3.0.

\section{Mathematical Modeling and Programming Algorithm Associated with Mathematical Simulation of The Volume of a Tank}

\begin{abstract}
This scientific article aims to present the results of an experience report of pedagogical actions for the resolution of a problem situation about the volume of a liquid varying inside a conical base cylindrical tank, which includes mathematical modeling and programming language specifically with regard to the possibility of interrelating two teaching techniques with a common purpose: mathematical modeling and programming algorithms associated with mathematical simulation. To achieve the objectives, we modeled mathematically the proposed problem situation with functions of two positive real variables and three sentences, using mathematical procedures and then mathematically simulate the values of the radius $\mathrm{R}$ and the depth " $\mathrm{d}$ " of the liquid inside and "outside" of the tank - directly in the developed computer program. The results of the mathematical and computational studies proved to be satisfactory to the objectives proposed for the studies, this is because, the values obtained by different methods presented the same real numbers.
\end{abstract}

Keywords: CBNC; MATLAB; VISUALG3.0.

Submetido em: 11/01/2021

Publicado em: 10/04/2021

\footnotetext{
${ }^{1}$ Mestre em Matemática Aplicada e Computacional pela UNICAMP. Professor do Curso de Licenciatura em Matemática da Universidade Federal do Amapá. Endereço para correspondência: Av. Metecos, 123 - Bairro: Renascer I, CEP: $68907-$ 210, Macapá, Amapá, Brasil. E-mail: joaoferreira@unifap.br.
} 


\title{
Algoritmo de Modelado Matemático Y Programación Asociado con La Simulación Matemática Del Volumen de un Tanque
}

\begin{abstract}
Resumen: Este artículo científico tiene como objetivo presentar los resultados de un relato de experiencia de acciones pedagógicas para la resolución de una situación problemática sobre el volumen de un líquido que varía dentro de un tanque cilíndrico de base cónica, que incluye modelado matemático y lenguaje de programación., Específicamente en lo que se refiere a la posibilidad de interrelacionar dos técnicas de enseñanza con un propósito común: modelado matemático y algoritmos de programación asociados a la simulación matemática. Para lograr los objetivos, primero modelamos matemáticamente la situación problema propuesta con funciones de dos variables reales positivas $\mathrm{y}$ tres oraciones, utilizando procedimientos matemáticos y luego simulamos matemáticamente los valores del radio $\mathrm{R}$ y la profundidad "d" del líquido en el interior y "fuera" del tanque - directamente en el programa informático desarrollado. Los resultados de los estudios matemáticos y computacionales resultaron satisfactorios a los objetivos propuestos para los estudios, esto se debe a que los valores obtenidos por diferentes métodos presentan los mismos números reales.
\end{abstract}

Palabras clave: BNCC; MATLAB; VISUALG3.0.

\section{Introdução}

O tema deste artigo científico versa sobre o relato de experiência de Modelagem Matemática ${ }^{2}$ da variação do volume de um líquido, no interior de um tanque sob a forma de um cilindro com base cônica, em que utilizamos algoritmo de programação e simulação matemática para exemplificar a aplicação de método numérico a estudantes da área de Engenharias e de Ciências Exatas e da Terra especialmente a licenciatura em Matemática. Também pode ser aplicado a alunos do ensino médio, principalmente com relação ao Itinerário Formativo de Matemáticas e suas Tecnologias, previsto na Base Nacional Comum Curricular (BNCC).

O objetivo visa estudar as dificuldades no processo de ensino-aprendizagem da Matemática e analisar o uso de modelagem matemática auxiliada por linguagem de programação para simular modelo de uma situação-problema proposta por Chapra (2013, p. 82-83) apresentando o detalhamento do modelo por meio de um arquivo-M no Matlab (2021), que o solucione.

Neste sentido, para tornar a aprendizagem lúdica e colaborativa, aproveitamos para programar primeiramente no software educacional Visualg (2021), pois oportuniza escrever os comandos em português, testar se a pseudo-linguagem ou portugol está logicamente escrita e se fornecerá os

\footnotetext{
${ }^{2}$ Esclarecemos ao leitor, que usamos a Modelagem Matemática como estratégia de ensino-aprendizagem de Matemática e, que por isso, alguns autores, como por exemplo, Biembengut e Hein (2000) a chamam de Modelação Matemática, pois ela, no processo educativo, não cumpre ou modificam consideravelmente as etapas do processo dela enquanto método de pesquisa. Tal fato, por exemplo, pode ser verificado na formulação do problema. Na Modelagem Matemática como método a formulação do problema se dá com o grupo social que tem um problema a ser resolvido, já enquanto método de ensino a formulação do problema se dá por uma mídia: televisão, jornal, livro, entre outros.
} 
resultados esperados corretamente. Depois destes procedimentos metodológicos, podemos convertêlos para o conjunto de códigos fontes do Matlab (2021), por isso propomos soluções viáveis para resolver o problema da aprendizagem dos estudantes.

Em seguida, realizamos a testagem de valores apresentados no enunciado do problema, ao efetuarmos analiticamente e simular computacionalmente os dados propostos no texto da situaçãoproblema, o que nos conduziu aos mesmos resultados.

Portanto, ao utilizar os modelos matemáticos, as estruturas de decisão para escrever o algoritmo e as simulações matemáticas, conseguiram resolver a situação-problema e os resultados estão apresentados neste texto.

\section{Referenciais Teórico e Metodológico}

Sobre o entrelaçamento de modelagem matemática e algoritmo de programação, para a resolução de situações-problema, Barcelos e Silveira (2012) defendem que o ensino de computação na educação básica deve fazer parte da formação escolar, não como uma técnica, mas sim como uma forma de organização de Pensamento Computacional e de resolução de situações-problemas. Esta ideia também é reforçada na BNCC, no que diz respeito à área de Matemática, onde as suas diretrizes estabelecem que: "Esses processos de aprendizagem são potencialmente ricos para o desenvolvimento de competências fundamentais para o letramento matemático (raciocínio, representação, comunicação e argumentação) e para o desenvolvimento do pensamento computacional.”. (BRASIL, 2018, p. 266).

É importante destacar que o pensamento computacional é um tema bem definido na BNCC: "pensamento computacional: envolve as capacidades de compreender, analisar, definir, modelar, resolver, comparar e automatizar problemas e suas soluções, de forma metódica e sistemática, por meio do desenvolvimento de algoritmos;" (BRASIL, 2018, p. 474) e incentivado a sua aplicação nas aulas de matemática, em destaque a interdisciplinaridade entre as unidades temáticas álgebra, geometria e probabilidade e estatística, conforme a seguir:

Outro aspecto a ser considerado é que a aprendizagem de Álgebra, como também aquelas relacionadas a Números, Geometria e Probabilidade e estatística, podem contribuir para o desenvolvimento do pensamento computacional dos alunos, tendo em vista que eles precisam ser capazes de traduzir uma situação dada em outras linguagens, como transformar situaçõesproblema, apresentadas em língua materna, em fórmulas, tabelas e gráficos e vice-versa. (BRASIL, 2018, p. 271).

Andrade et al. (2013, p. 170), definem o Pensamento Computacional: “[...] é um método para solução de problemas baseado nos fundamentos e técnicas da Ciência da Computação (Apud Wing, 2006)" e o seu uso no ensino e aprendizagem na educação básica, de maneira geral tem em “[...] Sua 
proposta e aplicar habilidades utilizadas para criação de programas computacionais como metodologia para resolução de problemas gerais.”. (ANDRADE et al., 2013, p. 170).

No artigo de Andrade et al. (2013, p. 170), são apresentadas três propostas de atividades de pensamento computacional, com a finalidade de explorar as etapas e apresentar instrumentos necessários, tais como: coleta, análise, representação de dados, abstração, discussão e conceitos necessários, ficha de coletas, execução do programa e análise dos resultados.

Carvalho e Klüber (2018, p. 2), em seus relatos de experiência em sala de aula, apresentam atividades a ser desenvolvida no software Scratch com o intuito de introduzir a programação computacional para alunos do ensino fundamental e nesse artigo, abordamos uma proposta semelhante de programação utilizando o Visualg (2021) e posteriormente o arquivo-M do Matlab (2021) para promover a prática proposta.

Nas Ciências Exatas e da Terra e nas Engenharias, a literatura indica que há quatro formas distintas de simulação: Simulação Icônica, Simulação Analógica, Simulação Matemática e Simulação Computacional. (RODRIGUES, 2012).

De acordo com Rodrigues (2012), a Simulação Matemática é aquela que usa conhecimentos básicos de matemática e física para modelar fenômenos e prever resultados. Esse método é o que o estudante normalmente usa - naturalmente e eventualmente sem perceber, por exemplo, quando dimensiona a variação do volume de um tanque em função do raio e da profundidade do líquido em seu interior sem que seja necessário utilizar instrumentos analógicos ou digitais para alcançar os seus objetivos.

Segundo Rodrigues (2012), os sistemas físicos utilizados na Simulação Matemática podem ser Mecânicos, Elétricos e Hidráulicos e a representação pode ser Diagramática como, por exemplo, a Figura 1 de Chapra (2013) e Matemática, como por exemplo, nas Equações (1) - (5) deste relato de experiência.

A BNCC estabelece que no ensino médio sejam ensinados objetos de conhecimento de forma aprofundada nas quatro áreas de conhecimento e este procedimento é denominado de Itinerário Formativo, que tem por finalidade estabelecer o seguinte:

Os itinerários formativos - estratégicos para a flexibilização da organização curricular do Ensino Médio, pois possibilitam opções de escolha aos estudantes - podem ser estruturados com foco em uma área do conhecimento, na formação técnica e profissional ou, também, na mobilização de competências e habilidades de diferentes áreas, compondo itinerários integrados, nos seguintes termos das DCNEM/2018 (BRASIL, 2018, p. 477, grifo do autor). são:

Em que os objetos de conhecimento do itinerário formativo de matemáticas e suas tecnologias,

II - matemática e suas tecnologias: aprofundamento de conhecimentos estruturantes para aplicação de diferentes conceitos matemáticos em contextos sociais e de trabalho, estruturando arranjos curriculares que permitam estudos em resolução de problemas e análises complexas, funcionais e não-lineares, análise de dados estatísticos e probabilidade, 
geometria e topologia, robótica, automação, inteligência artificial, programação, jogos digitais, sistemas dinâmicos, dentre outros, considerando o contexto local e as possibilidades de oferta pelos sistemas de ensino; (BRASIL, 2018, p. 477, grifo nosso).

Neste sentido, a simulação matemática aqui apresentada pode ser estendida aos alunos do ensino médio, porque utilizamos algoritmo de programação para testagem e validação dos modelos produzidos, interessados no itinerário formativo com o objetivo de prepará-los para a educação superior nas áreas de ciências exatas e da terra e de engenharias. A própria BNCC recomenda que sejam estimulados aos educandos que exerçam a atividade de modelagem matemática com o intuito de estimular a construção do pensamento algébrico, conforme a seguir:

A unidade temática Álgebra, por sua vez, tem como finalidade o desenvolvimento de um tipo especial de pensamento - pensamento algébrico - que é essencial para utilizar modelos matemáticos na compreensão, representação e análise de relações quantitativas de grandezas e, também, de situações e estruturas matemáticas, fazendo uso de letras e outros símbolos. (BRASIL, 2018, p. 270, grifo do autor).

Nas competências específicas de Matemática e suas tecnologias para o ensino médio da BNCC, a de número três (3), propõem aos professores e estudantes a utilizarem de estratégias, definições e procedimentos matemáticos para interpretar, construir modelos e resolver problemas em diversos contextos, analisando a plausibilidade dos resultados e a adequação das soluções propostas, de modo a construir argumentação consistente.

Nesse sentido, as atividades intelectuais da Modelagem Matemática, segundo Bassanezi (2002, p. 26-31), que podem subsidiar o planejamento são as seguintes: 1. Experimentação (o texto da situação-problema traz consigo os resultados hipotéticos desta etapa), 2. Abstração (a. Seleção de variáveis, b. Problematização ou formulação dos problemas teóricos numa linguagem própria da área em que se está trabalhando, c. Formulação de hipóteses, e d. Simplificação), 3. Resolução, 4. Validação (testagem dos dados nos modelos encontrados) e 5. Modificação (caso os resultados não reflitam o fenômeno observado).

Bassanezi (2002, p. 45 - 53) explica que a formulação de problemas deva ser o ponto de partida para a Modelagem Matemática, pois deverá ser bem planejada e fundamentada de modo que o modelo final reflita a realidade que se está estudando ou pesquisando; para que isso possa ocorrer, a escolha de temas, a coleta de dados e a formulação de modelos devem estar em consonância e intrinsecamente ordenados, percorrendo as trajetórias estabelecidas no fluxograma. No nosso caso, a formulação do problema é descrever o fenômeno da variação do volume do líquido no interior do tanque.

Segundo Almeida, Silva e Vertuan (2012) destacam que a Modelagem Matemática é descrita em termos de uma situação inicial (problemática), de uma situação final desejada (que representa uma solução para a situação inicial) e de um conjunto de procedimentos e conceitos necessários para passar da situação inicial para a situação final. Dessa forma, os autores segmentam esse conjunto de 
procedimentos em quatro fases: inteiração, matematização, resolução, interpretação de resultados e validação.

\section{Resultados do Estudo}

A situação-problema que motivou estes estudos encontra-se em Chapra (2013, pp. 82-83). É o livro de Métodos Numéricos Aplicados com MATLAB ${ }^{\circledR}$ para Engenheiros e Cientistas - utilizado na disciplina Cálculo Numérico para acadêmicos de Engenharia Elétrica da UNIFAP e de outras universidades. O enunciado da situação-problema é o seguinte:

3.1 A Figura P3.1 mostra um tanque cilíndrico de base cônica. Se o nível do líquido for muito baixo na parte cônica, o volume será simplesmente o volume cônico de líquido; se o nível do líquido estiver no meio da parte cilíndrica, o volume total de líquido incluirá a parte cônica preenchida e a parte parcialmente ocupada do cilindro.

Use estruturas de decisão para escrever um arquivo-M a fim de calcular o volume do tanque como função dos valores de $\mathrm{R}$ e de $\mathrm{d}$. Desenvolva a função de modo que ela retorne o volume para todos os casos em que a profundidade é menor que 3R. Retorne uma mensagem de erro ("Transbordo") se você transbordou o tanque, isto é, d $>3 R$. Teste a função com os seguintes dados:

Observe que o raio do tanque é $\mathrm{R}$.

Figura 1 - Figura P3.1

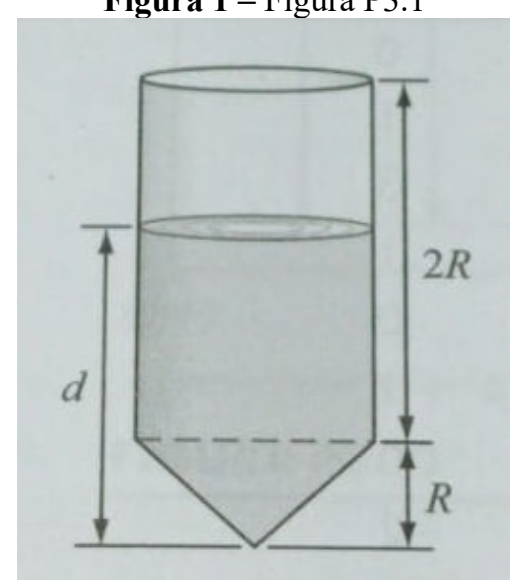

Fonte: Chapra (2013, p. 83).

Para solucionar este problema, a partir desta subseção, vamos aplicar as cinco etapas de modelagem matemática definidas por Basanezzi (2002, p. 26-31) para que se atinjam as metas solicitadas.

\subsection{Experimentação}

Segundo Bassanezi (2002, p. 26), a experimentação "É uma atividade essencialmente laboratorial onde se processa a obtenção de dados". Nesse artigo iniciaremos o processo de modelagem matemática a partir da abstração visto que o trabalho apresenta uma situação-problema advinda de referencial teórico (CHAPRA, 2013). 


\title{
3.2 Abstração
}

\section{a) Seleção das variáveis}

A seleção das variáveis ocorreu à medida que se buscava estabelecer os modelos e será também usada para declarar as variáveis reais no algoritmo de programação. Estão definidas conforme a seguir:

R: raio do tanque cilíndrico de base cônica;

d: altura ou profundidade da coluna de água;

VLC: volume do líquido somente na parte Cônica $(d<R)$;

VC: volume do cone $(d=R)$;

VTrC: volume do tronco do cone;

VLCC: volume do líquido no Cone e parte do Cilindro $(\mathrm{d}<=3 \mathrm{R})$;

VCi: volume do cilindro.

VLT: volume do líquido em que d > 3R;

VTC: volume do tanque cilíndrico de base cônica, i.e., o volume do cilindro mais o volume do cone $(d=3 R)$;

VT: volume do líquido que transborda $(\mathrm{d}-3 \mathrm{R})$.

\section{b) Problematização}

Estabelecida as dez (10) variáveis - sendo duas independentes e oito dependentes - da situação-problema que se está estudando, a Problematização vem a ser o segundo passo importante para se construir a modelagem matemática, por isso é imprescindível que:

\begin{abstract}
A adequação de uma investigação sistemática, empírica e crítica levam à formulação de problemas com enunciados que devem ser explicitados de forma clara, compreensível e operacional. Desta forma, um problema se constitui em uma pergunta científica quando explicita a relação entre as variáveis ou fatos envolvidos no fenômeno. (BASSANEZI, 2002, p. 28).
\end{abstract}

Deste modo, a situação-problema a ser trabalhado em nossa pesquisa é quais os modelos matemáticos que fornecem o volume do tanque cilíndrico-cônico em função das variáveis independentes $\mathrm{R}$ e d?

\section{c) Formulação de hipóteses}

H1: O volume do líquido da base cônica $(\mathrm{d} \leq \mathrm{R})$ é igual à diferença do volume do cone $(\mathrm{VC})$ e o volume do tronco do cone $(\mathrm{VTrC})^{3}$ :

$$
\mathrm{VLC}=\mathrm{VC}-\mathrm{VTrC} .
$$

\footnotetext{
${ }^{33}$ Justificando-se que escolhemos trabalhar com a diferença cone pelo tronco de cone para que os estudantes pudessem rememorar esses dois modelos. Sendo, então, uma escolha didática.
} 
H2: O volume do cone mais parte do volume do cilindro $(R<d \leq 3 R)$ é igual a soma do volume da base cônica (VC) com a parte do volume do cilindro (VCi):

$$
\mathrm{VLCC}=\mathrm{VC}+\mathrm{VCi} \text {. }
$$

H3: O volume de líquido maior de que o volume do tanque (VLT), quando d $>3 R$, é a soma do volume do tanque cilíndrico de base cônica (VTC), ou seja, quando $d=3 \mathrm{R}$, com o volume do líquido que transborda (VT):

$$
\mathrm{VLT}=\mathrm{VTC}+\mathrm{VT}
$$

H4: Para a construção do modelo matemático, na parte do cone, considerar a altura igual ao raio.

H5: Assumiremos Pi $(\pi)$ igual a 3,141593.

\section{d) Simplificação}

Conforme Bassanezi (2002, p. 29), devido a complexidade dos fenômenos, a simplificação do problema é importante para solucioná-lo, por isso, o texto da situação-problema foi simplificado sob a forma da Figura 1, com essa simplificação tornou-se mais fácil deduzir as funções a partir de teorias da geometria espacial. Escrever as funções referentes às hipóteses anteriores, também é uma forma de simplificação da situação-problema proposta, por isso, a partir da subseção a seguir apresentaremos os modelos matemáticos.

\subsection{Resolução}

\section{a) Escrever as funções que expressam o modelo matemático da situação-problema:}

De acordo com a variação de $R$ e d, isto é, $v=f(R, d)$, onde $v$ é o volume, $R$ é o raio do tanque e $\mathrm{d}$ a profundidade do líquido no tanque, o domínio e o contradomínio da função $\mathrm{v}=\mathrm{f}(\mathrm{R}, \mathrm{d})$ é a superfície definida por:

$$
\begin{gathered}
f: \mathbb{R}_{+}^{*} \times \mathbb{R}_{+}^{*} \mapsto \mathbb{R}_{+}^{*} \\
(R, d) \rightarrow v=f(R, d)
\end{gathered}
$$

em que $R, d \in \mathbb{R}_{+}^{*}$.

Ao desenvolver (1) temos o volume do líquido da base cônica (VLC):

$$
V L C=f_{1}(R, d)=\frac{\pi R^{3}}{3}-\frac{\pi(R-d)\left(R^{2}+d R+d^{2}\right)}{3}, \text { para } 0<d \leq R .
$$

Ao trabalharmos (2) temos o volume do líquido na parte cilíndrico-cônica (VLCC) é:

$$
V L C C=f_{2}(R, d)=\frac{\pi R^{3}}{3}+\pi R^{2}(d-R), \text { para } R<d \leq 3 R .
$$

Explorando (3) encontramos o volume do tanque cilíndrico de base cônica (VTC):

$$
V T C=\frac{7 \pi R^{3}}{3}, \text { para } d=3 R .
$$


O volume de líquido maior de que o volume do tanque (VLT), que provoca o transbordo é:

$$
V L T=f_{3}(R, d)=\frac{7 \pi R^{3}}{3}+\pi R^{2}(d-3 R), \text { para } d>3 R,
$$

O volume do líquido que transborda (VT) é medido pela segunda parcela da Equação (7), destacado abaixo:

$$
V T=\pi R^{2}(d-3 R) .
$$

Com as Equações (4) - (7) modelamos matematicamente o problema do tanque cilíndrico com base cônica, através da função definida por mais de uma sentença.

$$
f(R, d)=\left\{\begin{array}{c}
\frac{\pi R^{3}}{3}-\frac{\pi(R-d)\left(R^{2}+d R+d^{2}\right)}{3}, \text { para } 0<d \leq R \\
\frac{\pi R^{3}}{3}+\pi R^{2}(d-R), \text { para } R<d \leq 3 R \\
\frac{7 \pi R^{3}}{3}+\pi R^{2}(d-3 R), \text { para } d>3 R .
\end{array}\right.
$$

O procedimento seguinte é definir ou declarar as variáveis que serão utilizadas no algoritmo de programação.

\section{b) Iniciar e finalizar a estrutura de decisão de lógica de programação:}

Para escrever o algoritmo vamos utilizar a estrutura de decisão: "Se ... então ...", se a proposição é verdadeira e "Senão ...", se é falsa. Neste caso, segundo Vilarim (2004, p. 64):

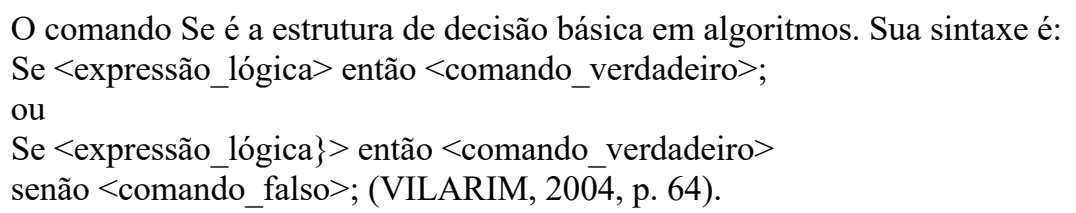

Essa estrutura da computação é a que mais adequada à formulação de uma lógica computacional que produza os resultados que se pretende.

Quadro 1 - Estruturas de decisão para escrever um arquivo no Visualg (2021) e no arquivo-M a fim de calcular o volume do tanque como função dos valores de $\mathrm{R}$ e de $\mathrm{d}$.

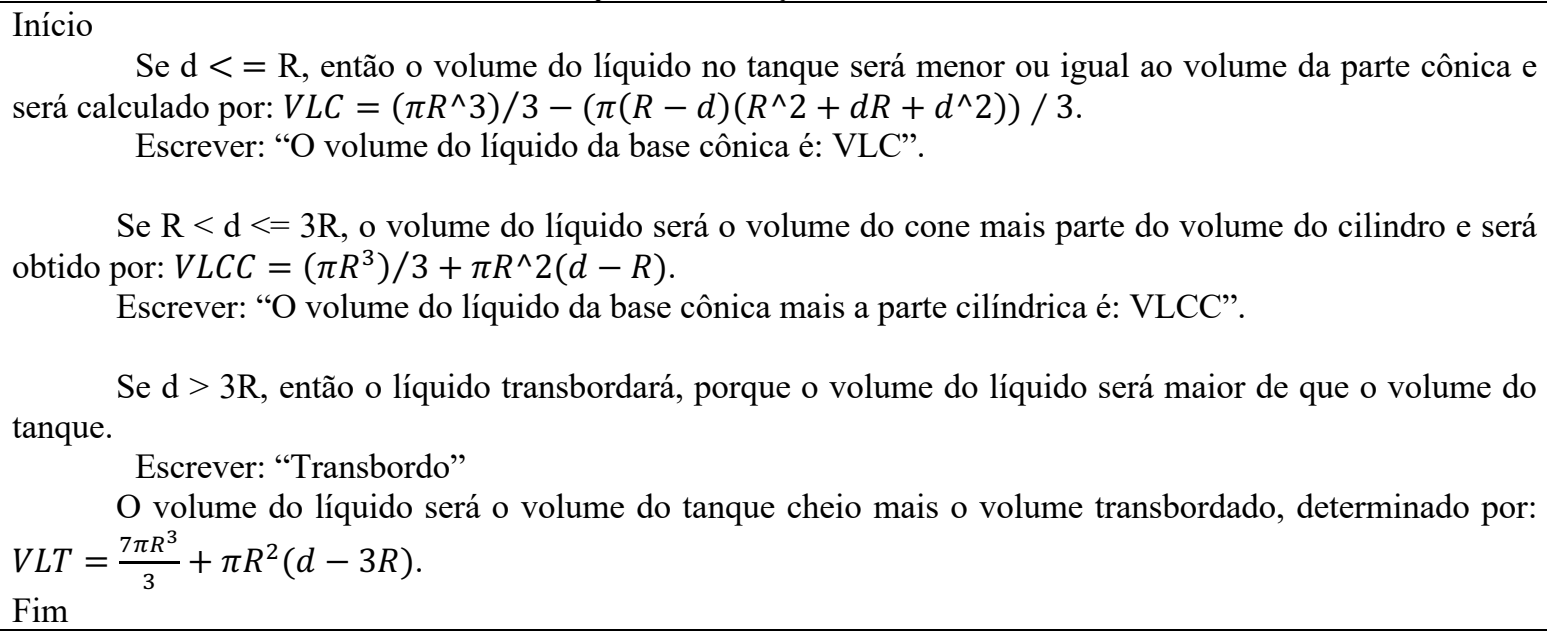

Se $\mathrm{R}<\mathrm{d}<=3 \mathrm{R}$, o volume do líquido será o volume do cone mais parte do volume do cilindro e será obtido por: $V L C C=\left(\pi R^{3}\right) / 3+\pi R^{\wedge} 2(d-R)$.

Escrever: "O volume do líquido da base cônica mais a parte cilíndrica é: VLCC".

Se d > 3R, então o líquido transbordará, porque o volume do líquido será maior de que o volume do tanque.

Escrever: "Transbordo"

$\mathrm{O}$ volume do líquido será o volume do tanque cheio mais o volume transbordado, determinado por: $V L T=\frac{7 \pi R^{3}}{3}+\pi R^{2}(d-3 R)$.

Fim

Fonte: elaborado pelo autor.

Nota: o símbolo <= significa "menor ou igual". 
c) Transcrever a estrutura de decisão do Quadro 1 para pseudo-linguagem portugol no Visualg (2021), conforme o Quadro 2 e posteriormente para o arquivo-M no Matlab (2021).

Quadro 2 - Pseudo-linguagem ou portugol do algoritmo para a resolução do problema envolvendo estrutura de decisão.

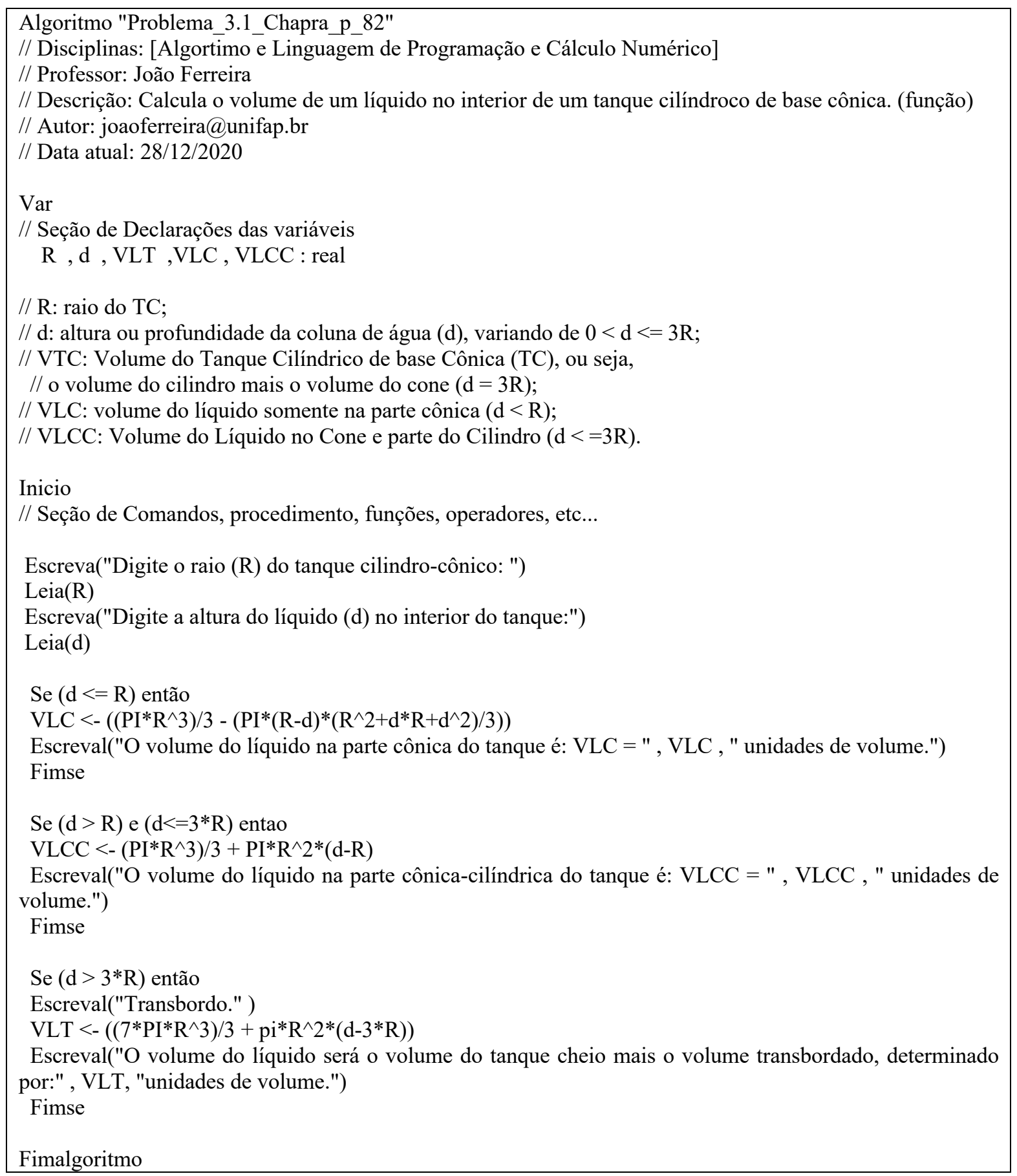

Fonte: elaborado pelo autor.

\subsection{Validação}

Em seguida, realizamos os testes para a tabela do texto de Chapra (2013), para validar o modelo.

a) Para $R=0,9$ e $d=1$, $(d>R)$, temos que o VLCC $=1,0179$ unidade de volume (u.v.). 
A Figura 1 mostra como os dados de entrada $\mathrm{R}$ e d são fornecidos ou inseridos ao programa e a saída é o volume do líquido.

Figura 2 - Compilação do programa no Visual (2021).

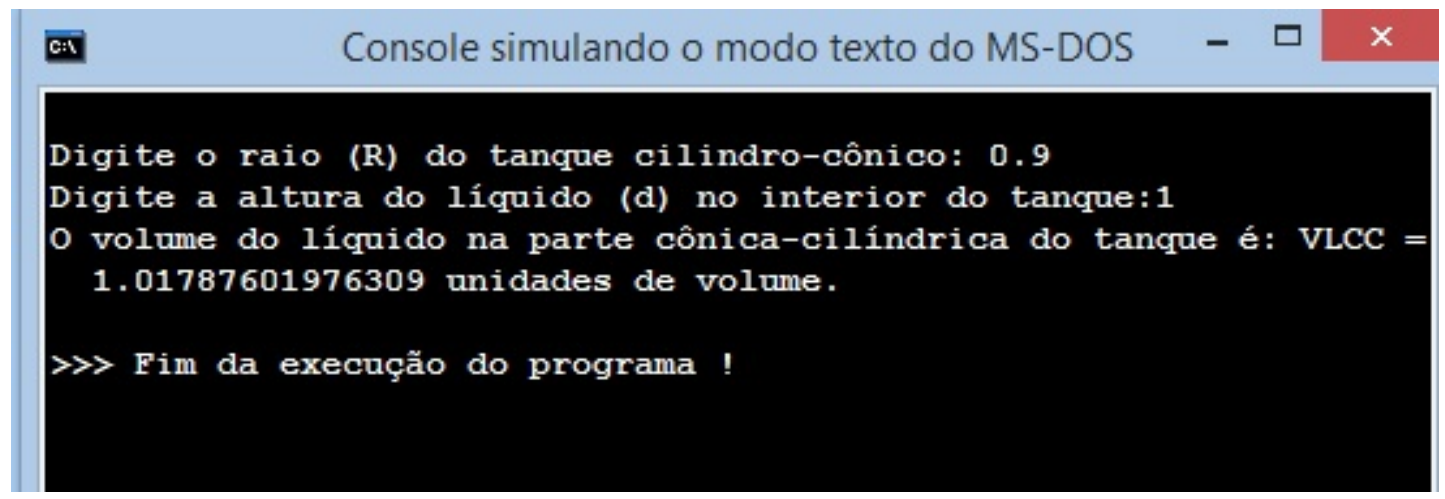

Fonte: elaborado pelo autor.

Como $\mathrm{R}<\mathrm{d}<3 \mathrm{R}$, o volume do líquido é menor de que o volume do tanque cilíndrico-cônico. O volume do tanque cilíndrico é calculado pela Equação (6):

$$
\begin{aligned}
V T C & =\left(7 \times 3.141593 \times(0.9)^{3}\right) / 3 \\
V T C & =16.03155 / 3 \\
V T C & =5.34285 \text { u.v. }
\end{aligned}
$$

Analiticamente, podemos determinar o valor obtido na Figura 2, substituindo-se os valores das variáveis computacionais $\mathrm{R}=0,9$ e $\mathrm{d}=1,0$ na Equação (5) e como $\mathrm{d}>\mathrm{R}$, vamos obter o volume de todo o cone e parte do cilindro de altura $(\mathrm{d}-\mathrm{R})$ :

$$
\begin{aligned}
& V L C C=\frac{\pi(0.9)^{3}}{3}+\pi(0.9)^{2}(1-0.9) \\
& V L C C=\frac{3.141593 \times 0.729}{3}+3.141593 \times 0.81 \times(0.1) \\
& V L C C=\frac{2.290221}{3}+3.141593 \times 0.081 \\
& V L C C=0,763407+0,254469 \\
& V L C C=1,017876 u . v .
\end{aligned}
$$

Este resultado é exatamente o determinado pelo programa conforme exibido na Figura 2. Esta verificação se faz necessária para que o programador (estudante ou acadêmico) sinta-se seguro na implementação do algoritmo de programação que desenvolveu.

\section{b) Para $R=1,5$ e $d=1,25$, $(d<R)$, temos que o $V L C=2,0453$ unidades de volume.}

Na Figura 3, encontra-se o resultado obtido pelo programa computacional.

Neste caso, o volume do tanque cilíndrico de base cônica é maior de que o volume do líquido em seu interior, que é de VTC $=24,74004$ unidades de volume, de acordo com os cálculos a seguir:

$$
V T C=\frac{7 \times 3,141593 \times(1.5)^{3}}{3}=
$$




$$
\begin{aligned}
& V T C=\frac{7 \times 3,141593 \times 3,375}{3}= \\
& V T C=\frac{74.22013}{3}=24,74004 u . v .
\end{aligned}
$$

Veja que o volume do líquido no tanque cilíndrico utilizando o Visualg (2021), é menor de que o $\mathrm{VTC}=24,74$ unidades de volume:

Figura 3 - Console do Visualg (2021) com o valor do volume

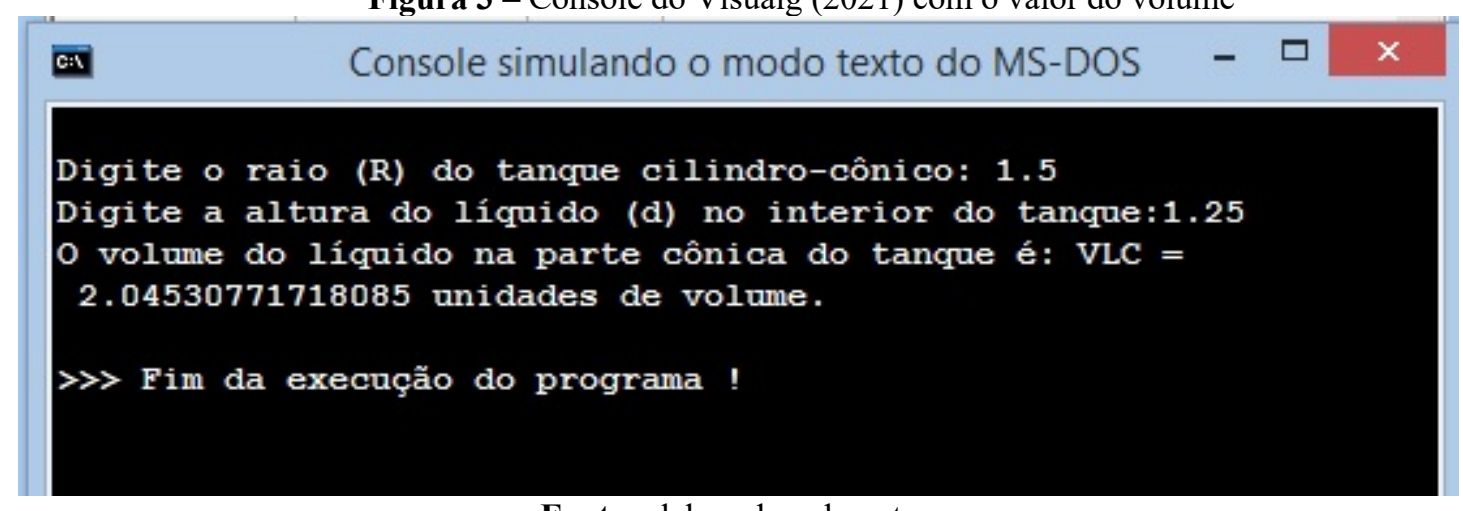

Fonte: elaborado pelo autor.

Algebricamente, substituem-se os valores das variáveis computacionais $R=1,5$ e $d=1,25$ na Equação (4) e como $d<R$, vamos determinar o volume de parte do cone de altura $(R-d)$ :

$$
\begin{aligned}
& V L C=\frac{\pi(1.5)^{3}}{3}-\frac{\pi(1.5-1.25)\left((1.5)^{2}+(1.25)(1.5)+(1.25)^{2}\right)}{3} \\
& V L C=\frac{3.141593 \times 3.375}{3}-\frac{3.141593(0.25)(2.25+1.875+1.5625)}{3} \\
& V L C=\frac{10.60288}{3}-\frac{0.785398(5.6875)}{3} \\
& V L C=3.534292-\frac{4.466953}{3} \\
& V L C=3.534292-1,4889884 \\
& V L C=2,045308 u . v .
\end{aligned}
$$

c) Para $R=1,3$ e d = 3,8 $(d>2 R)$, temos que $V L C C=15,5739$ unidades de volume.

$\mathrm{Na}$ Figura 4, observa-se que os valores do raio e da profundidade (altura) do líquido no interior do tanque são digitados e o volume é calculado pelo programa. 
Figura 4 - Resultado no console do Visualg (2021), para o caso em que $d>2 R$.

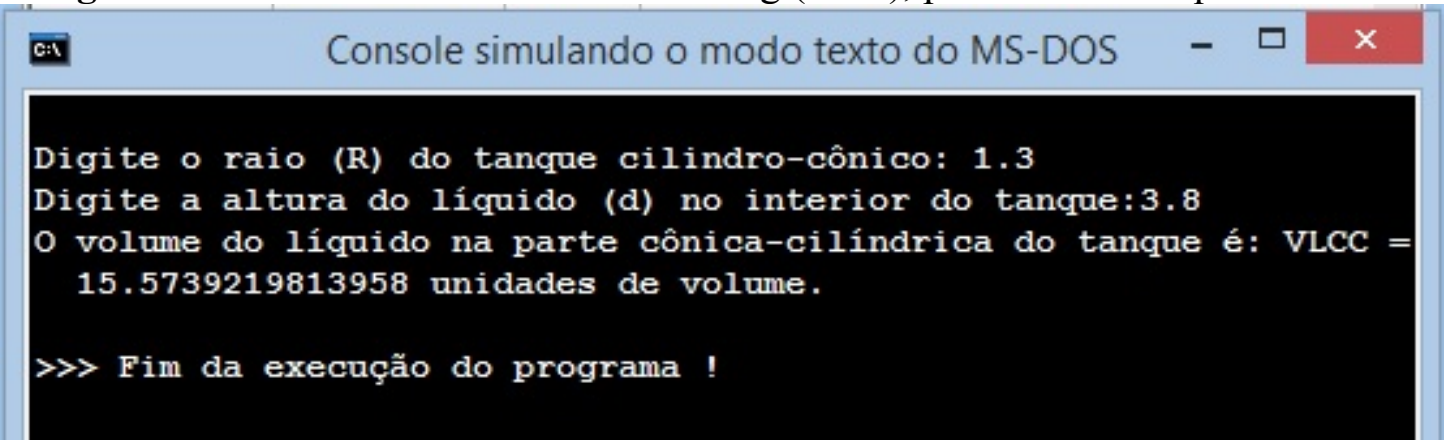

Fonte: elaborado pelo autor.

Para verificar se o resultado apresentado pelo programa está correto, vamos substituir $\mathrm{R}=$ 1.3 e $\mathrm{d}=3.8$ na Equação (5), porque $\mathrm{d}>2 \mathrm{R}$ :

$$
\begin{aligned}
& V L C C=\frac{\pi(1.3)^{3}}{3}+\pi(1.3)^{2}(3.8-1.3) \\
& V L C C=\frac{3.141593 \times 2.197}{3}+3.141593 \times 1.69 \times(2.5) \\
& V L C C=\frac{6.90208}{3}+5.309292 \times(2.5) \\
& V L C C=2.300693+13.27323 \\
& V L C C=15.57392 u . v .
\end{aligned}
$$

Este resultado com cinco casas decimais confere com o realizado pelo programa computacional desenvolvido para a simulação matemática.

d) Para $R=1,3$ e d = 4.0, (d > 3R), temos que o líquido transbordará.

Figura 5 - Resultado apresentado pelo Visualg (2021).

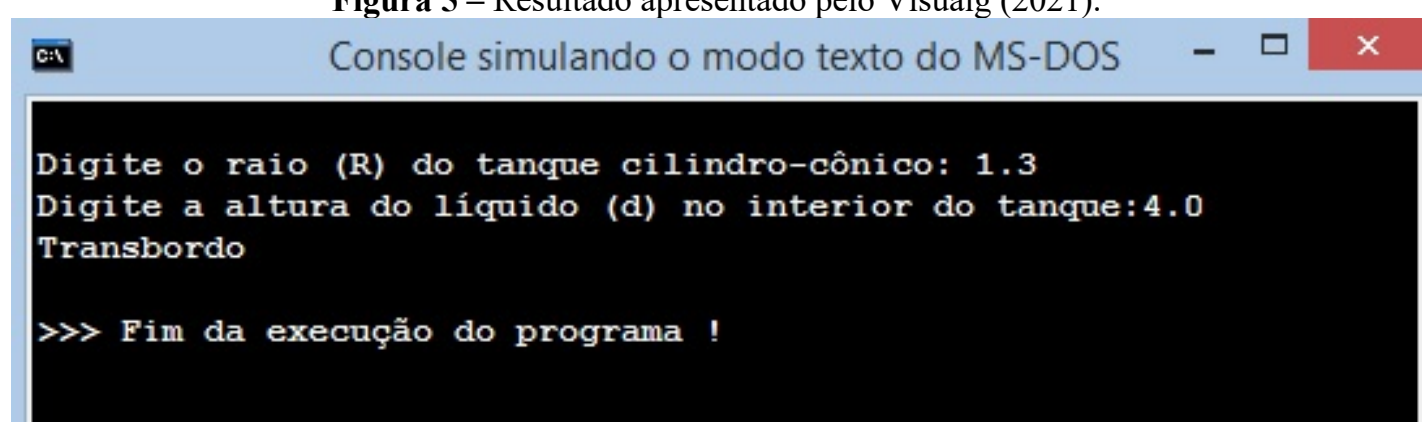

Fonte: elaborado pelo autor.

Como $d>3 R$, vamos substituir os dados $R=1,3$ e $d=4,0$ na Equação (7) para obter o volume do líquido que é maior de que o volume do tanque:

$$
\begin{aligned}
& V L T=\frac{7 \pi R^{3}}{3}+\pi R^{2}(d-3 R) \\
& V L T=\frac{7 \times 3.141593 \times(1.3)^{3}}{3}+3.141593 \times(1.3)^{2}(4.0-3 \times 1.3) \\
& V L T=\frac{21.99115 \times 2.197}{3}+3.141593 \times 1.69 \times(4.0-3.9)
\end{aligned}
$$




$$
\begin{aligned}
& V L T=\frac{48.31456}{3}+5.309292 \times(0.1) \\
& V L T=16.10485+0.5309292 \\
& V L T=16.63578 \text { u.v. }
\end{aligned}
$$

Este resultado é maior de que o volume do tanque cheio, que é de aproximadamente 16.10485 unidades de volume.

\section{e) Arquivo-M no Matlab (2021)}

O algoritmo com a estrutura de repetição do Quadro 1 foram transcritos para os códigos fontes do Matlab (2021) conforme registros no Quadro 3:

Quadro 3 - Código fonte ou Arquivo-M do Matlab (2021).

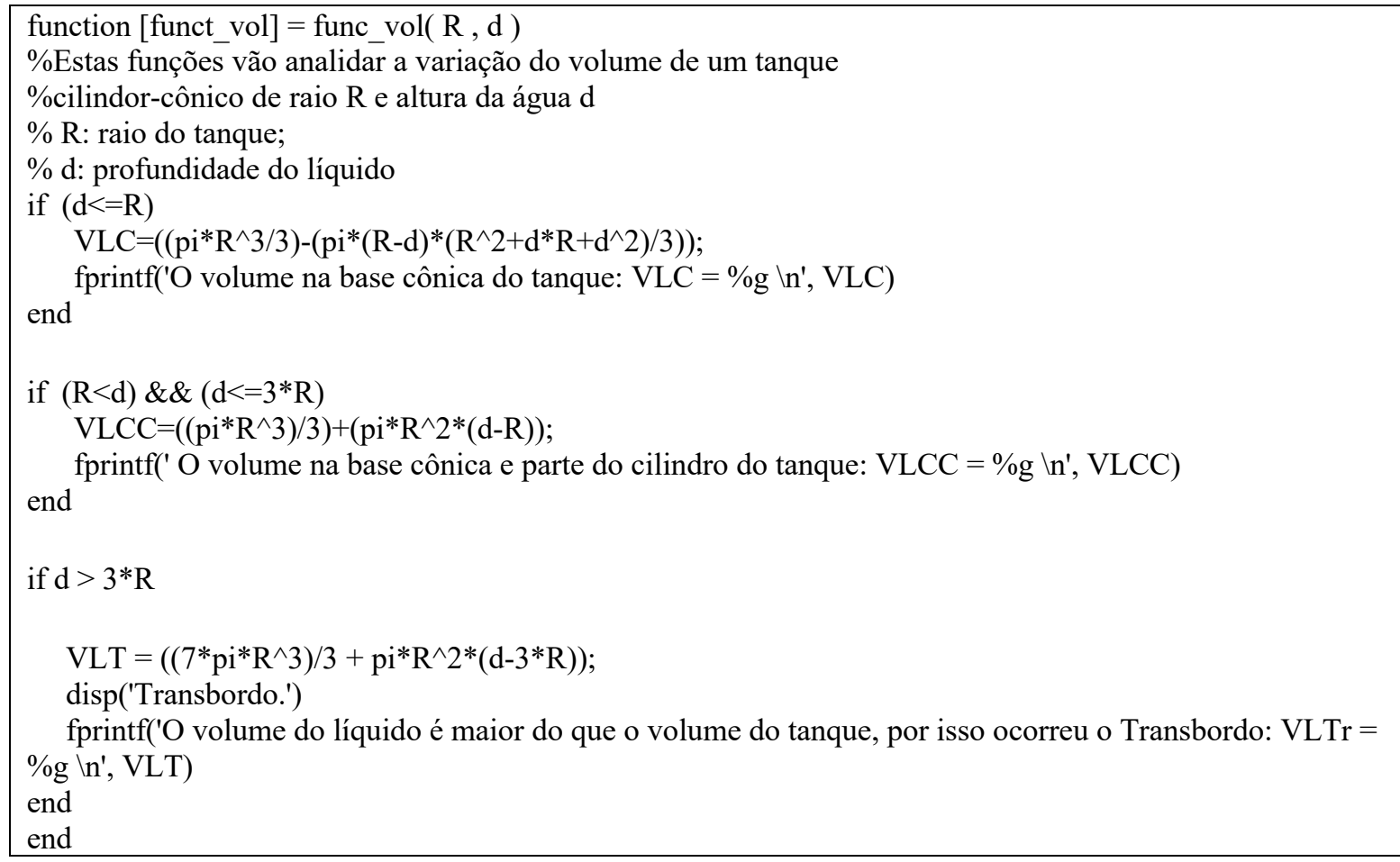

Fonte: elaborado pelo autor.

Substituindo-se os valores de R e d no programa do Quadro 3, no Matlab (2021), obtemos os mesmos resultados apresentados nas Equações (10) - (15). No Quadro 4, estão as inserções das quatro simulações do problema, com os resultados iguais aos obtidos pelo aplicativo Visualg (2021).

Quadro 4 - Reprodução da janela de Command Window do Matlab (2021).

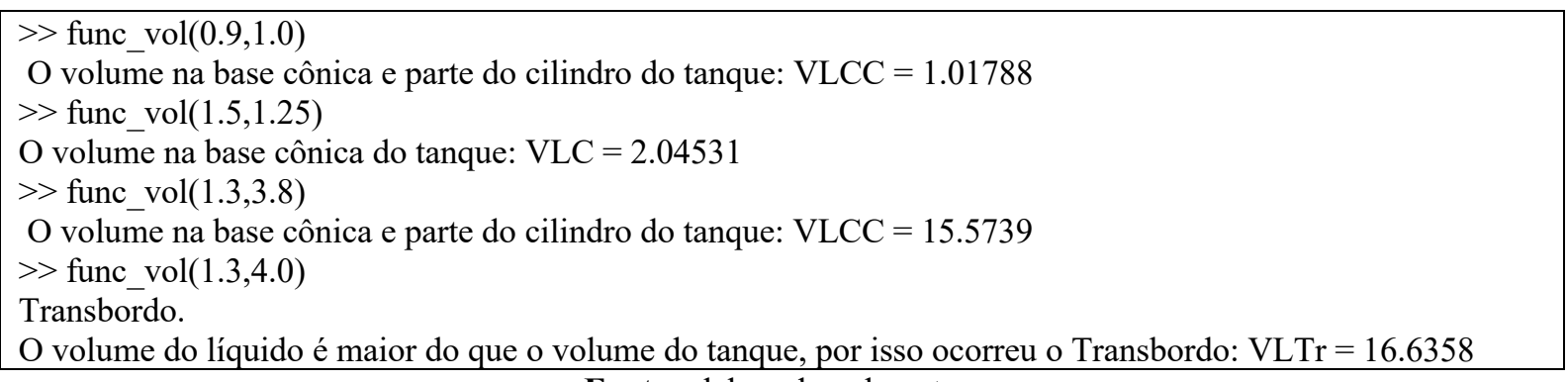

Fonte: elaborado pelo autor. 


\subsection{Modificação}

Após diferentes abordagens na resolução do modelo matemático do problema chegamos a resultados análogos comprovando a eficiência dos pseudocódigos e a possibilidade de aplicação desta metodologia, de tal modo que foi possível perceber a importância de proporcionar ao estudante a oportunidade de utilizar a pesquisa matemática como meio de aprendizagem. Portanto, não há necessidade de se modificar o modelo matemático.

\section{Considerações finais}

A Modelagem Matemática como forma de ensino tem sido alvo de muitos estudos e pesquisas em todo o mudo. No Brasil, já vem ocorrendo a mais de trinta anos. Vários modos de utilizá-la em sala de aula têm sido propostos a fim de proporcionar melhor compreensão e interação de professores e estudantes envolvidos no processo. Nesse sentido, percebe-se que começa a destacar-se o método pedagógico denominado Pensamento Computacional (do ingles, computational thinking), sinalizado como alternativa metodológica para esse fim que tem como direcionamento o trabalho com modelos matemáticos construídos a partir de objetos de conhecimento pré-existentes que serão analisados, aplicados e servirão de guia para o estudo do conteúdo curricular.

O algoritmo desenvolvido neste processo de ensino e aprendizagem mostrou-se satisfatório às simulações matemáticas da situação-problema apresentada por Chapra (2013, p. 82-83), pois os dados da tabela do enunciado do problema foram testados computacionalmente e analiticamente e os resultados se confirmaram, conforme as simulações numéricas apresentados nas Figuras 2 - 5, que foram executados pelo Visualg (2021) e posteriormente os cálculos matemáticos expressos nas respectivas Equações (10) - (15), com o propósito de verificar se o algoritmo estava correto.

Nos baseamos na sequência lógica do Quadro 1 para escrever em portugol no Visualg (2021) e na linguagem de máquina do arquivo-M do Matlab (2021), conforme solicitado no enunciado da situação-problema. A simulação matemática é muito importante para estudar o comportamento de um objeto ou fenômeno, porque possibilita que se estude determinado fenômeno sem a necessidade de construir o modelo físico ou tangível - neste caso o tanque cilíndrico-cônico. Sendo assim, os acadêmicos de interessados terão a possibilidade de conhecer como ocorre esta prática e que poderá ser utilizada por eles quando estiver em seu ambiente de trabalho profissionalmente.

Com a recente implantação da BNCC, abrem-se novas fronteiras para a produção científica e lançamento de novos produtos didáticos-pedagógico para o apoio a experiências curriculares inovadoras, por isso, estamos diante de inúmeras possibilidades de propor soluções viáveis para resolver o problema da aprendizagem dos estudantes. 


\section{Referências}

ALMEIDA, L. M. W.; SILVA, K. P.; VERTUAN, R. E. Modelagem matemática na educação básica. São Paulo: Contexto, 2012.

ANDRADE, Daiane et al. Proposta de atividades para o desenvolvimento do pensamento computacional no ensino fundamental. Anais do Workshop de Informática na Escola, [s.1.], p. 169, nov. 2013. ISSN 2316-6541. Disponível em: https://brie.org/pub/index.php/wie/article/view/2645/2299. Acesso em: 25 fev. 2021. doi:http://dx.doi.org/10.5753/cbie.wie.2013.169.

BARCELOS, T. S.; SILVEIRA, I. F. Pensamento computacional e educação matemática: relações para o ensino de computação na educação básica. In: WORKSHOP SOBRE EDUCAÇÃO EM COMPUTAÇÃO, 20., 2012, Curitiba. Anais do XXXII CSBC. 2012. p. 23. Disponível em: https://www.researchgate.net/profile/Thiago-

Barcelos/publication/256439343 Pensamento Computacional e Educacao Matematica Relacoes para_o_Ensino_de_Computacao_na_Educacao_Basica/links/0deec5228dfbb4d377000000/Pensame nto-Computacional-e-Educacao-Matematica-Relacoes-para-o-Ensino-de-Computacao-naEducacao-Basica.pdf. Acesso em: 24 fev. 2021.

BASSANEZI, C. R. Ensino-aprendizagem com modelagem matemática. 3. ed. Campinas, SP: UNICAMP, 2002. https://www.researchgate.net/publication/256007243 Ensino_aprendizagem_com_Modelagem_matematica. Acesso em: 09 dez. 2020.

BRASIL. Ministério da Educação (MEC). Base Nacional Comum Curricular (BNCC): educação é a base. Brasília: MEC, 2018. Disponível em: http://basenacionalcomum.mec.gov.br/wpcontent/uploads/2018/12/BNCC_19dez2018_site.pdf. Acesso em: 15 dez. 2020.

CARVALHO, F. J.; KLÜBER, T. E. Modelagem matemática aliada à programação de computadores na educação básica. In: ENCONTRO PARANAENSE DE MODELAGEM NA EDUCAÇÃO MATEMÁTICA, 8., 2018, Cascavel - PR. Anais do VIII EPMEM. Cascavel, PR: 2018. Disponível em:

http://sbemparana.com.br/eventos/index.php/EPMEM/VIII_EPMEM/paper/viewFile/820/418. Acesso em: 24 fev. 2021.

CHAPRA, S. C. Métodos numéricos aplicados com MATLAB ${ }^{\circledR}$ para engenheiros e cientistas. 3. ed. Porto Alegre: AMGH, 2013. p. 82-83.

BIEMBENGUT, M. S.; HEIN, N. Modelagem matemática no ensino. Blumenau: Ed. Contexto, 2000.

MATLAB. Software de programação e cálculo numérico. Disponível em:

https://la.mathworks.com/products/matlab.html. Acesso em: 06 jan. 2021.

RODRIGUES, D. Tipos de simulação. E aí, convergiu. [s.1.], 04 set. 2012. Disponível em: https://eaiconvergiu.wordpress.com/2012/09/04/tipos-de-simulacao/. Acesso em: 23 fev. 2021.

VILARIM, G. Algoritmos: programação para iniciantes. 2. ed. Rio de Janeiro: Editora Ciência Moderna Ltda., 2004. 
VISUALG. Compilador de portugol. Disponível em: http://visualg3.com.br/baixe-o-visualg-3-07/. Acesso em: 06 jan. 2021. 